\title{
REVIEW ARTICLE OPEN \\ Loss of oral mucosal stem cell markers in oral submucous fibrosis and their reactivation in malignant transformation
}

\author{
Mohit Sharma (D) ${ }^{1}$, Felipe Paiva Fonseca ${ }^{2}$, Keith D. Hunter ${ }^{3}$ and Raghu Radhakrishnan ${ }^{4}$
}

The integrity of the basal stem cell layer is critical for epithelial homoeostasis. In this paper, we review the expression of oral mucosal stem cell markers (OM-SCMs) in oral submucous fibrosis (OSF), oral potentially malignant disorders (OPMDs) and oral squamous cell carcinoma (OSCC) to understand the role of basal cells in potentiating cancer stem cell behaviour in OSF. While the loss of basal cell clonogenicity triggers epithelial atrophy in OSF, the transition of the epithelium from atrophic to hyperplastic and eventually neoplastic involves the reactivation of basal stemness. The vacillating expression patterns of OM-SCMs confirm the role of keratins 5, 14, 19, CD44, $\beta 1$-integrin, p63, sex-determining region $Y$ box (SOX2), octamer-binding transcription factor 4 (Oct-4), c-MYC, B-cell-specific Moloney murine leukaemia virus integration site 1 (Bmi-1) and aldehyde dehydrogenase 1 (ALDH1) in OSF, OPMDs and OSCC. The downregulation of OM-SCMs in the atrophic epithelium of OSF and their upregulation during malignant transformation are illustrated with relevant literature in this review.

International Journal of Oral Science (2020)12:23 ； https://doi.org/10.1038/s41368-020-00090-5

\section{INTRODUCTION}

The basal stem cell layer of normal oral mucosa (NOM) is a selfperpetuating reservoir of cells with a mechanism for self-renewal, a property referred to as clonogenicity or stemness. The integrity of the basal stem cell layer is thus essential for epithelial homoeostasis. Breakdown in cell-cycle turnover is antecedent to the development of oral potentially malignant disorders (OPMDs) and oral squamous cell carcinoma (OSCC). Oral submucous fibrosis (OSF) is an OPMD commonly present among people in the Indian subcontinent and Southeast Asia. ${ }^{1,2}$ Various epidemiological studies implicate areca nut chewing as the main aetiological factor in OSF. There is overwhelming evidence suggesting that the chewing of commercial addictive products, such as pan masala, gutka, mawa and betel quid (BQ) containing considerable amounts of areca nut, tobacco and slaked lime, predisposes patients to OSF., ${ }^{1,3}$ Areca nut has cytotoxic effects on oral mucosal cells, ${ }^{4}$ and disturbingly, oral cancer arising in the background of OSF seems to develop earlier and has a greater propensity to invade and metastasize. ${ }^{1}$

Considering OSF as an over-healing wound, the role of stem cell activity in its genesis is well documented. ${ }^{2,5,6}$ Several reports suggest downregulated basal stem cell activity as a tipping event triggering epithelial atrophy in OSF.,7-14 Limited scientific evidence supports a rebound amplification of stem cell activity in the epithelium transitioning from atrophic OSF to oral epithelial dysplasia (OED) and eventually to OSCC. A comprehensive assessment of oral mucosal stem cell markers (OM-SCMs) in relation to the progression of OSF, OED and OSCC is performed in this review (Figs. 1-5).

\section{STEMNESS REGULATION: THE ROLE OF WILD-TYPE VERSUS MUTATED P53}

When mutated, p53 triggers a cascade of events leading to malignancy. However, its role in OSF and its malignant transformation are not clear. Since p53 antibodies (e.g., p53-duo) do not distinguish between wild-type p53 (Wt-p53) and mutated p53 (Mut-p53), it is critical to delineate their role in the progression of OSF.

Wt-p53 expression seems to be vital for the initiation of fibrosis to the extent that the expression of profibrotic plasminogen activator inhibitor-1 (PAI-1) is re-established following the expression of Wt-p53. ${ }^{15}$ Transforming growth factor-beta (TGF- $\beta$ ) induces the complex formation between Wt-p53 and Smads2/3/4 in the PAI-1 promoter, recruiting the histone acetyltransferase CREBbinding protein (CBP). CBP augments histone $\mathrm{H} 3$ acetylation in the PAl-1 promoter, activating PAl-1 transcription. ${ }^{16}$ Thus, Wt-p53 is expressed intensely in the basal layer of the atrophic epithelium in OSF compared to the hyperplastic epithelium, ${ }^{13}$ suggesting that Wt-p53 plays a key role in the initiation of fibrosis and epithelial atrophy by reducing stemness.

Wt-p53 represses stemness by inducing miR-145, ${ }^{17}$ which exerts tumour-suppressor functions through the downregulation of c-MYC, octamer-binding transcription factor 4 (Oct-4) and sexdetermining region Y-box 2 (SOX2). ${ }^{17,18}$ Notably, atrophic epithelium in OSF shows high p53 levels, low c-MYC expression and stable hypoxia-inducible factor (HIF) expression. ${ }^{13}$ The clonal expansion and evolution of dysplasia is the outcome of high c-MYC activity and Mut-p53 expression. ${ }^{13}$ The downregulation of Oct- 4 in the atrophic epithelium of OSF in contrast to the normal

\footnotetext{
${ }^{1}$ Department of Oral Pathology, Sudha Rustagi College of Dental Sciences and Research, Faridabad, Haryana, India; ${ }^{2}$ Department of Oral Surgery and Pathology, School of Dentistry, Federal University of Minas Gerais, Belo Horizonte, MG, Brazil; ${ }^{3}$ Academic Unit of Oral and Maxillofacial Medicine and Pathology, School of Clinical Dentistry, University

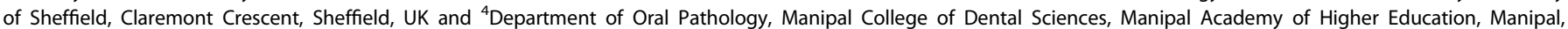
Karnataka, India

Correspondence: Raghu Radhakrishnan (raghu.ar@manipal.edu)
}

Received: 25 January 2020 Revised: 26 June 2020 Accepted: 29 June 2020

Published online: 21 August 2020 

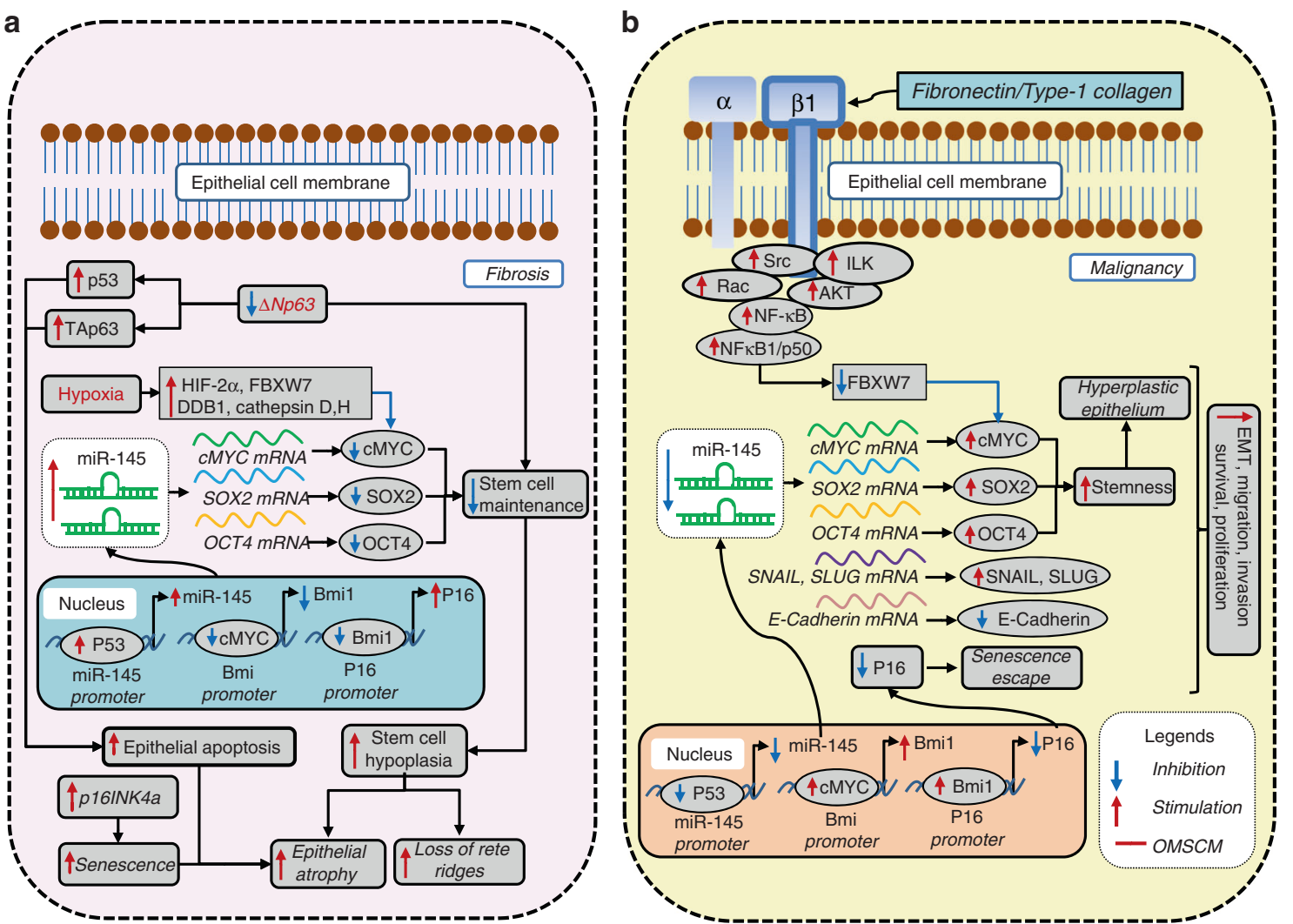

Fig. 1 c-MYC, SOX2 and OCT-4 as oral mucosal stem cell markers (OM-SCMs) in oral submucous fibrosis (OSF). a Their downregulation mediates epithelial atrophy, and $\mathbf{b}$ their upregulation mediates malignancy

epithelium ${ }^{4}$ (Fig. 1a) and its rebound expression in the malignant transformation of OSF suggests altered stemness (Fig. 1b). ${ }^{19}$ Thus, it could be concluded that Wt-p53 works as an anti-stemness factor and is associated with fibrosis and atrophy, while Mut-p53 is associated with dysplasia and malignant progression. ${ }^{20}$

\section{ALTERATIONS IN THE EXPRESSION PATTERN OF OM-SCMS IN OSF, OPMD AND OSCC}

The OM-SCs in the basal layer of the oral mucosa are the normal stem cells essential for maintaining the integrity of the oral mucosa. ${ }^{21}$ Contact with the basement membrane is required to maintain basal keratinocyte stemness. The severity of the contact of OM-SCs with the basement membrane promotes their differentiation. ${ }^{22}$ Their biological attributes, such as inherent longevity and the ability to self-replicate, make these cells an ideal candidate to accumulate a full complement of mutations triggering tumorigenesis. Hence, their breakdown is reflected in the aberrant expression of OM-SCMs in various OPMDs and OSCC. Although the stem cells associated with cancer are quite similar to normal stem cells and express the same markers, certain unique characteristics, such as the loss of growth control, justify their separate designation as cancer stem cells (CSCs) or tumourinitiating cells. ${ }^{21,23,24}$

Several studies have shown that CSCs play a crucial role in the growth, spread and recurrence of oral cancer. ${ }^{25-31}$ CSCs in oral cancer demonstrate elevated expression levels of stem cell markers, such as Oct-4, SOX2, Nanog, Nestin, CK19, B-cellspecific Moloney murine leukaemia virus integration site 1 (Bmi1), CD117 (c-kit), CD44 and CD133, and decreased expression levels of involucrin and CK-13. ${ }^{25,26,32-34}$ Evidence supporting this stems from the finding that the signalling pathways regulating normal stem cell division (i.e., Notch, Wnt, Hedgehog and Bmi-1) are involved in oral cancer development. . $7,30,31,35,36^{\text {Recent }}$ evidence indicates that CSCs within surgical margins play a crucial role in the clinical outcomes of cancer. ${ }^{37-39}$ Lazarevic et al. ${ }^{38}$ showed that the surgical margins of oral cancer express stem cell markers, such as CD44, Oct-4, CD133, Nanog and SOX2, which have the ability to form spheroids to become resistant to chemotherapy. CSCs survive and promote cancer, as they maintain low levels of reactive oxygen species (ROS) levels and exhibit redox patterns matching those of normal stem cells. ${ }^{23,40}$ This explains their resistance to elimination by radiotherapy. ${ }^{23,40}$

$\mathrm{Xu}$ et al. $^{41}$ showed that enhanced aerobic glycolysis and L-lactate production in oral CSCs is mediated via the epidermal growth factor (EGF)/epidermal growth factor receptor (EGFR)/ phosphoinositide 3-kinase (PI3K)/HIF-1a pathway, and is evidenced by the upregulation of the $\mathrm{CD} 44^{+} \mathrm{CD} 24^{-}$population of CSCs along with the expression of other CSC markers, such as Bmi- 1 and aldehyde dehydrogenase 1 (ALDH1). Zhao et al. ${ }^{42}$ showed that the cancer stem cell-like state in oral cancer is primed by lactate uptake, and is evidenced by the expression of CD133 and upregulated Wnt signalling.

Individually, ALDH1 and CD44 are considered markers of CSCs in oral cancer, ${ }^{31,43}$ and together, they identify the specific CSC compartment. ${ }^{43-46}$ ALDH1 was found to be upregulated at the invasive tumour front (ITF), and the adjacent non-tumour epithelium correlated with tumour aggressiveness. ${ }^{47}$ Aldehyde dehydrogenase 1 family member $A 1$ (ALDH1A1) may thus serve as a marker for premalignant cells in oral cancer. ${ }^{48}$ CD44 high/ ALDH $1{ }^{\text {high }}$ cells exhibit a greater tumour sphere-forming capability than $\mathrm{CD} 44^{\text {high }} / \mathrm{ALDH} 1^{\text {low }}$ cells. ${ }^{46}$ Remarkably, vimentin-positive spindle-shaped cells were found in the CD44 $4^{\text {high }} / A L D H 11^{\text {high }}$ cell population but not in the $\mathrm{CD} 44^{\text {high }} / \mathrm{ALDH} 1^{\text {low }}$ cell population, ${ }^{46}$ indicating the propensity of the former to undergo epithelial-mesenchymal transition (EMT) and seed new tumours. 


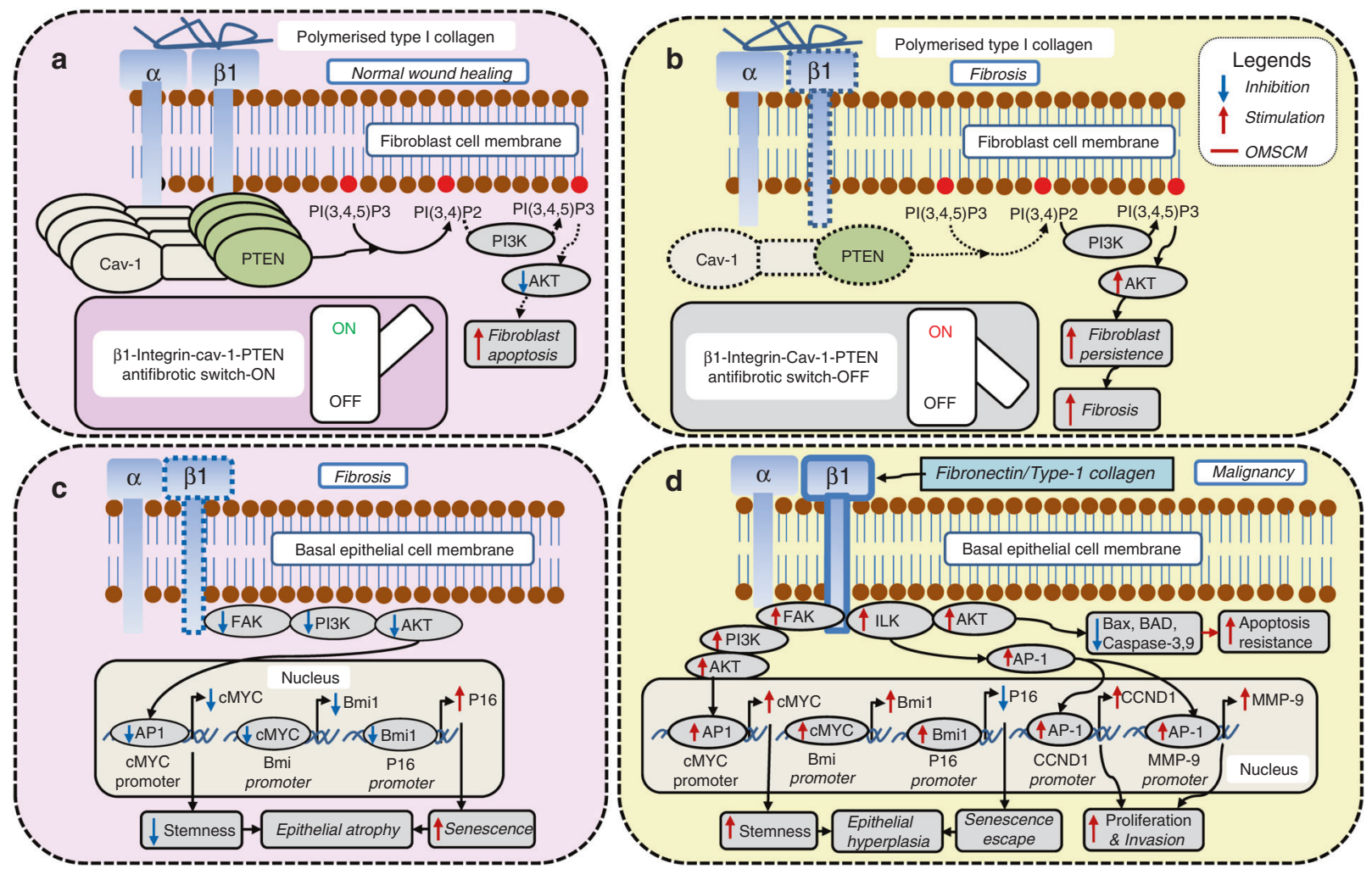

Fig. $2 \beta_{1}$-integrin as an oral mucosal stem cell marker (OM-SCM) in oral submucous fibrosis (OSF). a In fibroblasts, normal $\beta_{1}$-integrin levels are antifibrotic, and $\mathbf{b}$ reduced $\beta_{1}$-integrin levels promote fibrosis. In epithelial cells, $\mathbf{c}$ decreased $\beta_{1}$-integrin levels promote epithelial atrophy, and $\mathbf{d}$ increased $\beta 1$-integrin levels promote malignancy

Certainly, the increased expression of vimentin correlates with higher migratory activity and oral cancer progression. ${ }^{39}$

Other stemness markers, such as CD44, CD133, glucoseregulated protein 78 (Grp-78), Grp-96, Oct-4, Nanog and SOX2, are upregulated upon exposure to areca nut and correlate with worse prognosis in areca nut-induced cancers. ${ }^{49,50}$ Furthermore, areca nut-induced chemoradioresistance results from the upregulation of ATP-binding cassette subfamily G member-2 (ABCG-2), a drug- efflux pump and a stem cell marker. ${ }^{22,49,50}$ Oral cancers among habitual areca nut chewers demonstrate an aggressive phenotype, chemoradioresistance and a much lower 5-year survival rate than those without areca habits. ${ }^{18}$

Studies on oral premalignant lesions have shown that CSC markers, such as ABCG-2 and Bmi-1, predict the transformation of oral leukoplakia to cancer. ${ }^{51}$ The expression patterns of CSC markers, such as ALDH1 and CD133, correlate with a high risk of malignant transformation of oral leukoplakia. ${ }^{52}$ In addition, the coexpression of the CSC markers ALDH1 and Bmi-1 is a strong indicator of malignant transformation of oral erythroplakia. ${ }^{20}$ It is well established that the CSC markers ALDH1, Bmi-1 and ABCG-2 drive the process of field cancerization in oral erythroplakia. ${ }^{53,54}$

An intriguing correlation of stem cell activity in OSF, OPMD and OSCC was uncovered through our literature search. While Rajendran et al. ${ }^{7}$ was the first to propose downregulated stem cell activity in OSF, it was subsequently confirmed to be due to the adverse effects of nitric oxide and areca nut-associated carcinogens on basal stem cells. ${ }^{11}$ The reduced stem cell activity in atrophic OSF was evidenced by a decrease in the expression of proliferative/stem cell markers, such as Ki-67, Cyclin-D1 (CCND-1) and $\mathrm{C}_{-M Y C}{ }^{10,13}$ and an increase in their expression in the malignant transformation of OSF. ${ }^{10,13}$

The OM-SCMs involved in molecular signalling pathways include keratins $5 / 14,15,19, a_{6} \beta_{4}$-integrin ${ }^{+} C D 71^{-}, \beta_{1}$-integrin, collagen IV, p75 ${ }^{\mathrm{NGFR}}$, stage-specific embryonic antigen 1 (SSEA1),
CD24, CD44 (CD44H), CD71, CD117 (c-kit), CD133, melanomaassociated chondroitin sulfate proteoglycan (MCSP), Nestin, p63, octamer-binding transcription factor-3/4 (Oct-3/4), Nanog, SOX2, ABCG-2, ALDH1 and Bmi-1 (Supplementary Information $(\mathrm{SI}))^{22,55-65}$ The crucial role of keratins $5,14,19, \mathrm{CD} 44, \beta 1$-integrin, p63, SOX2, Oct-4, c-MYC, Bmi-1 and ALDH1 in potentiating stem cell behaviour in OSF is further discussed in sections " $\beta 1$-integrin as a stem cell marker in OSF and its malignant evolution", "p63 as a stem cell marker in OSF and its malignant transformation", "cMYC as a stem cell marker in OSF and its malignant evolution", "Bmi-1 is a stem cell marker in OSF and its malignant evolution", "Keratin $5 / 14$ as a stem cell marker in OSF and its malignant evolution" and "Keratin-19 as a stem cell marker in OSF and its malignant evolution".

$\beta 1$-integrin as a stem cell marker in OSF and its malignant evolution

$\beta_{1}$-integrin functions as OM-SCM since it is downregulated in differentiated cells. The modulation of collagen synthesis during wound healing occurs via $\beta_{1}$-integrin. Polymerized collagen inhibits an excessive accumulation of ECM by activating $\beta 1$ integrin. ${ }^{9,66}$ When fibroblasts interact with polymerized type I collagen, caveolin-1 (Cav-1) forms a complex with phosphatase and tensin homologue (PTEN) and $\beta 1$-integrin on the plasma membrane. ${ }^{66}$ This places PTEN in the correct spatial location to inhibit the PI3K/AKT signal generated through the $\beta_{1^{-}}$ integrin-matrix interaction, ${ }^{66}$ thus promoting fibroblast apoptosis (Fig. 2a). Conversely, the downregulation of $\beta 1$-integrin leads to reduced membrane accumulation of the PTEN-Cav-1- $\beta_{1}$-integrin complex. $^{9,66}$ This hampers the ability of PTEN to inactivate AKT signalling, ${ }^{66}$ leading to fibroblast persistence and promoting fibrosis (Fig. 2b).

Hyperplastic OSF and OSCC demonstrate increased stem cell activity, as evidenced by the upregulation of $\beta_{1}$-integrin. ${ }^{9}$ An 

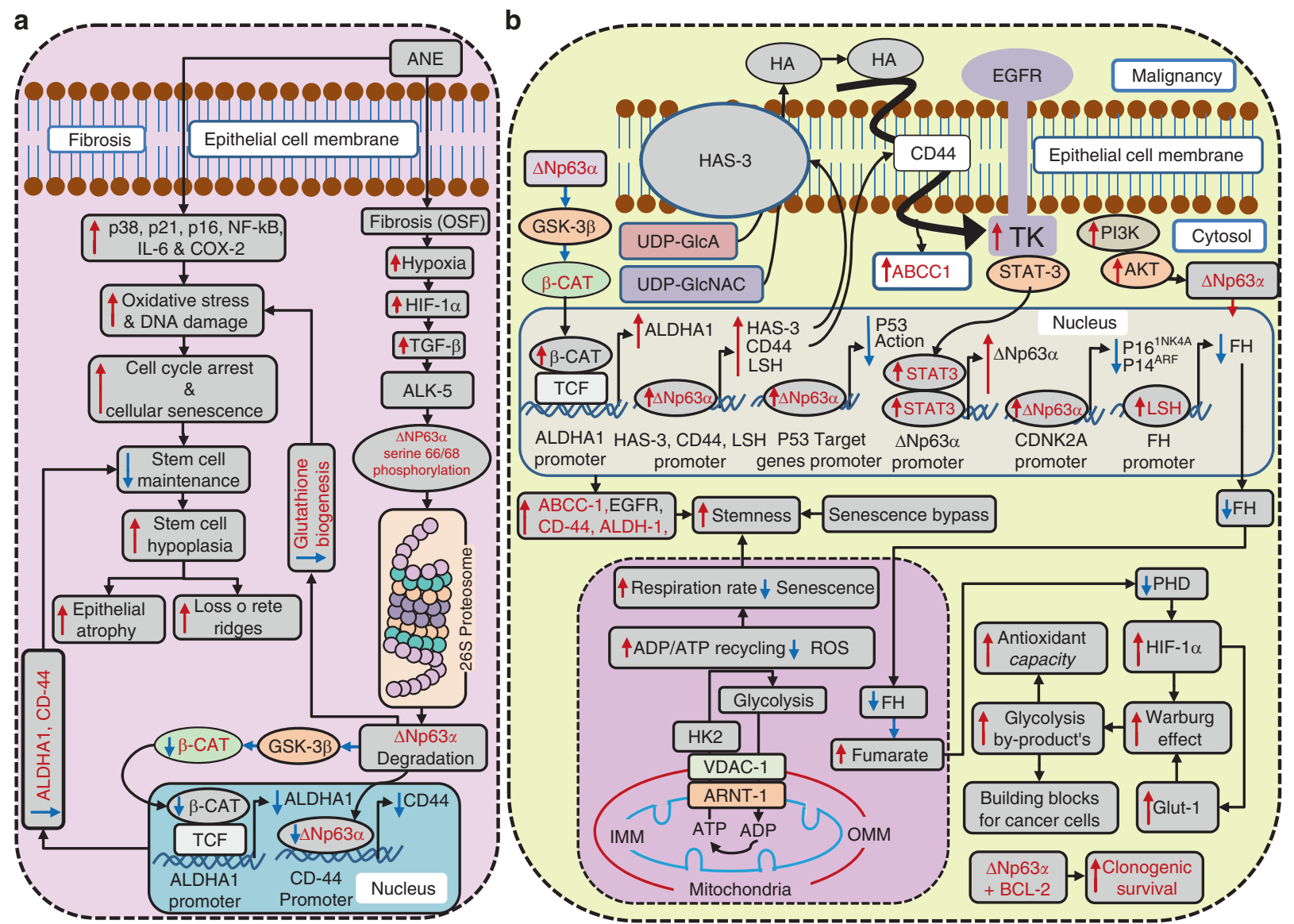

Fig. $3 \Delta \mathrm{Np63 \alpha}$ as an oral mucosal stem cell marker (OM-SCM) in oral submucous fibrosis (OSF). a Its downregulation mediates epithelial atrophy, and $\mathbf{b}$ its upregulation mediates malignancy

increased $\beta 1$-integrin expression pattern correlates with a greater tumorigenic potential, as indicated by the augmented tumour spheres and holoclonal colony formation in oral cancer cells compared to control cells. ${ }^{67}$ The $\beta_{1}$-integrin-driven transition from dormancy to tumorigenicity occurs through its activation by fibronectin/type-1 collagen and/or the activation of uPA receptor (uPAR). ${ }^{68} \beta 1$-integrin, through the integrin-linked kinase (ILK)/AKT pathway, inhibits various proapoptotic enzymes, such as BCL-2associated $\mathrm{X}$ apoptosis regulator (BAX), BCL-2-associated agonist of cell death (BAD) and caspase-3/-9, and thereby promotes apoptosis resistance in cancer cells. ${ }^{69}$ Increased ILK also upregulates CCND-1, which promotes cellular proliferation, and matrix metalloproteinase-9 (MMP-9), promoting the invasive potential. ${ }^{69}$ $\beta 1$-integrin also upregulates c-MYC through the focal adhesion kinase (FAK)/PI3K/AKT pathway, ${ }^{69,70}$ resulting in increased stemness and hyperplastic epithelium in OSF (Fig. 2d). Conversely, the downregulation of $\beta 1$-integrin in the epithelial compartment should lead to an atrophic epithelium. Certainly, very advanced OSF with a severely atrophic epithelium does show the lowest stem cell activity, evidenced by the downregulation of $\beta_{1}$-integrin in buccal mucosa (Fig. 2c). ${ }^{9}$

p63 as a stem cell marker in OSF and its malignant transformation The p63 gene products occur in six different protein isoforms, of which $\Delta N p 63 a, \Delta N p 63 \beta$ and $\Delta N p 63 \gamma$ are devoid of the N-terminal transactivation domain, whereas TAp63a, TAp63 $\beta$ and TAp63 $\gamma$ act as transcription factors. Among these, $\triangle \mathrm{Np} 63 \mathrm{a}$ is the predominant isoform whose expression is reduced in OSF without OED and increased in OSF with severe OED. ${ }^{12,14}$ The expression of $\triangle \mathrm{Np} 63 \mathrm{a}$ confirms the regenerative potential, as it is restricted to the basal stem cell layer in NOM. ${ }^{61}$ Downregulated $\Delta$ Np63a mediates senescence, ${ }^{61,71}$ which is considered to be a barrier to tumour development. Oxidative stress and DNA damage, which mediate cell-cycle arrest and the senescence of keratinocytes in OSF following exposure to areca nut extract (ANE), are a result of the overactivity of p16, p21, p38, nuclear factor kappa B (NF-KB), IL-6 and COX-2 (Fig. 3a). ${ }^{72}$

$\Delta$ Np63a functions as a dominant-negative inhibitor of $\mathrm{p} 53$, competing with its DNA-binding sites to promote oral cancer. ${ }^{73}$ Thus, the downregulation of $\triangle \mathrm{Np} 63 \mathrm{a}$ activates $\mathrm{Wt}-\mathrm{p} 53{ }^{15}$ further contributing to apoptosis and epithelial atrophy in OSF. Likewise, $\Delta$ Np63a inhibits TAp63, which mirrors p53 function. ${ }^{12,74}$ If $\Delta N p 63 a$ is reduced, the unhampered TAp63 mediates apoptosis and epithelial atrophy in OSF (Fig. 1a).

TGF- $\beta_{1}$-TGF $\beta$ R1 (ALK-5)-mediated serine-66/68 phosphorylation of the $\Delta \mathrm{Np} 63 \mathrm{a}$ isoform induces $26 \mathrm{~S}$ proteasomal degradation (Fig. 3a). ${ }^{61}$ Impaired $\Delta$ Np63a isoform function through TGF- $\beta_{1}{ }^{61}$ might be responsible for oxidative stress-mediated epithelial atrophy in OSF, as reported by Khan et al. ${ }^{4}$ and Wang et al. ${ }^{75}$ (Fig. 3a). Indeed, $\Delta$ Np63a has the ability to inhibit the cell death induced through oxidative stress, DNA damage and anoikis via the upregulation of glutathione biogenesis, and it cooperates with B-cell lymphoma 2 (BCL-2) to promote clonogenic survival (Fig. 3a, b). ${ }^{75}$ Since $\triangle N p 63 a$ is a positive regulator of OM-SCMs, ALDH1 and CD44, its downregulation ${ }^{61,76}$ is reflected in impaired basal stem regeneration (Fig. $3 a) .^{61,76}$ The decreased stem cell maintenance manifests as stem cell hypoplasia, epithelial atrophy and loss of rete ridges (Fig. $3 a$ ).

The augmentation of $\Delta \mathrm{Np} 63 \mathrm{a}$ in keratinocytes mediates senescence bypass through the chromatin- remodelling protein lymphoid-specific helicase (Lsh) and endows them with selfrenewal abilities, indicated by the presence of a $\mathrm{K}-15^{+}$stem cell 
population. ${ }^{77}$ In addition, $\Delta$ Np63a promotes the repression of p16 $6^{\text {Ink4a }}$ and p19 ARF, especially in the presence of retrovirusassociated DNA sequencing (RAS), thus promoting malignant transformation (Fig. 3b). ${ }^{58,71,77}$

Recently, the mechanism by which $\Delta N p 63$ maintains stem cell potential has been elucidated. ${ }^{78} \Delta$ Np63 augments hexokinase- 2 (HK-2) expression by binding to the p63-binding motif in the 15th intronic region of the HK-2 genomic sequence, which works as an enhancer. ${ }^{78} \mathrm{HK}-2$, via its mitochondrial-binding motif (MBF), affixes voltage-dependent anion channel 1 (VDAC-1) in the outer mitochondrial membrane (OMM, red). ${ }^{78}$ VDAC- 1 in turn interacts with adenine nucleotide translocase-1 (ANT-1) located in the inner mitochondrial membrane (IMM, blue), forming a channel between IMM and OMM. ${ }^{78,79}$ This mechanism allows rapid ADP/ATP cycling through increased coupling between glycolysis and OXPHOS.78 Augmented coupling between glycolysis and OXPHOS protects cells from oxidative stress by reducing ROS formation ${ }^{79}$ and thereby preventing senescence. ${ }^{80}$ This allows for higher respiratory rates and increases the stem cell capacity (Fig. 3b). ${ }^{78,79}$

In addition, $\Delta$ Np63a-arbitrated lymphoid-specific helicase (Lsh) upregulation $^{77}$ can epigenetically suppress fumarate hydratase (FH), a Krebs cycle enzyme. ${ }^{81}$ This leads to the accumulation of fumarate, considered an oncometabolite, which then inhibits the inhibitors of hypoxia-inducible factor (HIF), the prolyl hydroxylases (PHDs). ${ }^{82}$ Consequently, amplified HIF can promote malignancy through the Warburg effect by providing cancer cells with building blocks (Fig. 3b). ${ }^{83}$

$\triangle \mathrm{Np63a}$ has been shown to be reactivated in OED and OSCC. ${ }^{14,78}$ Upregulated $\triangle N$ p63 promotes oral cancer chemoresistance and proliferation by activating EGFR, multidrug resistanceassociated protein 1 (MRP1)/ATP-binding cassette subfamily $C$ member 1 (ABCC-1), ALDH1 and CD44 (Fig. 3b). ${ }^{76}$ A pervasive signalling network promoting stemness in oral cancer exists, involving $\triangle N p 63 a$, hyaluronan synthase 3 (HAS-3), hyaluronic acid $(\mathrm{HA}), \mathrm{CD} 44, \mathrm{EGFR}$ and signal transducer and activator of transcription-3 (STAT-3). ${ }^{76} \Delta$ Np63a binds to the HAS-3 and CD44 promoter, upregulating their expression and promoting their translocation to the cell membrane. ${ }^{76,84}$ Membranous HAS-3 promotes the intracellular synthesis of $\mathrm{HA}$ from precursors uridine diphosphate $\mathrm{N}$-acetylglucosamine (UDP-GIcNAc) and uridine diphosphate-glucuronic acid (UDP-GICA), and then $H A$ is extruded out of the cell membrane. ${ }^{84}$ The exteriorized HA then binds to its receptor CD44, which transactivates EGFR. ${ }^{76}$ Activated EGFR through STAT-3 upregulates $\Delta N p 63 a^{85} \Delta$ Np63a has STAT-3binding elements in its promoter, fulfilling this purpose (Fig. 3b). ${ }^{86}$ This mechanism explains the attainment of stem cells in oral cancer via EGF. $\triangle N$ p63a indirectly upregulates ALDHA1 through the $\Delta \mathrm{Np} 63 \mathrm{a} / \mathrm{GSK}-3 / \beta$-catenin ( $\beta$-CAT)/ALDHA1 pathway (Fig. $3 \mathrm{~b}$ and Supplementary Information). ${ }^{62,63}$

c-MYC as a stem cell marker in OSF and its malignant evolution Stromal hypoxia is an essential factor in the pathogenesis of OSF, ${ }^{2,13,14}$ and is mediated through several pathways. ${ }^{2}$ Hypoxiainduced enzymes, such as Cathepsin-D, H and E3 ubiquitin ligases such as F-box and WD-repeat domain containing 7 (FBXW-7) and DNA damage-binding protein 1 (DDB-1) can promote the proteolysis and proteasomal degradation, respectively, of the cMYC protein. ${ }^{87}$ This can result in the loss of proliferative potential and epithelial atrophy. Similarly, the downregulation of c-MYC through hypoxia-induced HIF-2a can decrease stemness, ${ }^{88}$ leading to atrophy in OSF. As discussed previously, the atrophic epithelium in OSF shows low c-MYC expression (Fig. 1a). ${ }^{13}$

Several studies have shown c-MYC to be a necessary positive modulator of the proliferative compartment. ${ }^{89,90}$ The nuclear expression of c-MYC in the basal and parabasal cells of NOM ascribes its proliferative potential. The loss of nuclear c-MYC expression in the differentiated layers of oral mucosa further substantiates its role in maintaining basal stemness. ${ }^{13}$ c-MYC overexpression in tumours has been attributed to multiple mechanisms, including stabilizing mutations, amplifications and chromosomal translocations. ${ }^{87}$ In addition, it is upregulated by $\beta 1-$ integrin through the FAK/PI3K/AKT pathway (Fig. 2d) ${ }^{69,70}$ and by NF-KB through the Src/Rac and integrin-linked kinase (ILK)/AKT pathways. ${ }^{69}$ Interestingly, the NF-KB1/p50 subunit of NF-KB has been shown to inhibit C-MYC protein degradation via its inhibition by the E3 ubiquitin ligase FBXW-7, ${ }^{91}$ thus leading to elevated c-MYC levels (Fig. 1b).

Oct-4 and SOX2 are upregulated due to the downregulation of miR-145 by arecoline. ${ }^{18}$ As miR-145 is a positive regulator of E-cadherin and a negative regulator of Snail (SNAI1) and Slug (SNAI2), EMT occurs. The exposed epithelial cells thus acquire increased chemoresistance, augmented migration, increased invasiveness and anchorage-independent growth. Furthermore, SOX2 and Oct-4 expression is inversely related to miR-145 expression in the tissues of individuals with areca quid-induced OSCC. ${ }^{18}$ Since miR-145 downregulates C-MYC, the suppression of miR-145 drives the upregulation of c-MYC (Fig. 1b). ${ }^{92}$

Bmi-1 is a stem cell marker in OSF and its malignant evolution Bmi-1 is positively regulated by c-MYC and increases cellular proliferation by suppressing the INK4a locus (Fig. 2d). ${ }^{93-95}$ Downregulated Bmi-1 can propagate epithelial atrophy in $\mathrm{OSF}^{71,77}$ via enhanced senescence (Fig. 2c). ${ }^{95}$

The $\beta 1$-integrin/FAK/PI3K/AKT/AP-1 pathway leads to the activation of $\mathrm{Bmi}-1^{96}$ through c-MYC (Fig. 2d). ${ }^{95}$ Whilst Bmi-1 expression is limited to the basal layer in normal epithelium, dysplastic and carcinomatous epithelium show Bmi-1 expression in the superficial layers. ${ }^{95}$

Importantly, the knockdown of Bmi-1 in normal epithelium did not cause an immediate arrest of replication or a loss of viable cells, whereas in OSCC cells, it had these two effects. ${ }^{95}$ In addition, the knockdown of Bmi-1 was shown to inhibit the tumourinitiating properties of $\mathrm{ALDH}^{+}{ }^{+}$cells, ${ }^{27,97}$ enhance their radiosensitivity and ${ }^{27,97}$ chemosensitivity ${ }^{27}$ and inhibit metastasis. ${ }^{97}$ On the other hand, the overexpression of Bmi- 1 alters $\mathrm{ALDH} 1^{-}$cells to become $\mathrm{ALDH}^{+}$cells, increasing tumour volume and enhancing metastatic foci. ${ }^{97}$ The coexpression of Bmi-1/Snail/ALDH1 correlates with the worst prognosis in oral cancer patients. ${ }^{97}$ Bmi-1 is thus considered to be a potential chemotherapeutic and radiotherapeutic target for increasing the sensitivity of CSCs. ${ }^{27}$

Keratin $5 / 14$ as a stem cell marker in OSF and its malignant evolution

Downregulated epithelial stemness via K-5/14 inhibition. Keratin 5/ 14 are normally expressed in basal-proliferating keratinocytes through activator protein $1 / 2$ (AP-1/2) or specificity protein 1,3 $(\mathrm{SP}-1,3)^{64}$ along with $\Delta \mathrm{Np} 63$. The expression of keratin $5 / 14$ is mediated by the binding of $\Delta \mathrm{Np} 63$ with AP-1,2 or SP-1,3 in the $\mathrm{K}-14$ enhancer. In addition, in the K-14 promoter, $\Delta \mathrm{Np} 63$ binds with AP2 to upregulate K-14 expression. In the K-5 promoter, $\triangle \mathrm{Np63}$ binds to upregulate its expression. ${ }^{61,65}$ The primary aetiological agent of OSF, ANE, ostensibly downregulates Keratin $5 / 14$ through TGF- $\beta 1$-mediated $\triangle N p 63$ abrogation, ${ }^{61,65}$ although it upregulates AP-1/2 and SP-1,3 (Fig. 4a). ${ }^{64}$

Regaining epithelial stemness through K-5/14 upregulation. EMT in OSF is mediated through the downregulation of membranous E-cadherin and $\beta$-CAT, as well as miR-205 and par-3 family cell polarity regulator (PARD-3), and the upregulation of $\mathrm{N}$-cadherin, Twist-1, Zinc finger E-box-binding homeobox 1 (ZEB-1), MMP-9 and Vimentin, a mesenchymal-specific protein and a tell-tale marker of EMT in epithelial cancers. ${ }^{12}$ Subsequently, the expression of vimentin, which is associated with some degree of fibrosis in $\mathrm{OSF}^{98}$ has been shown to upregulate keratin 5/14 through $\Delta \mathrm{Np63}$ in OSCCs. ${ }^{65}$ Even $\Delta$ Np63 itself promotes EMT, ${ }^{12}$ which is shown to promote stemness (discussed later). It has been 

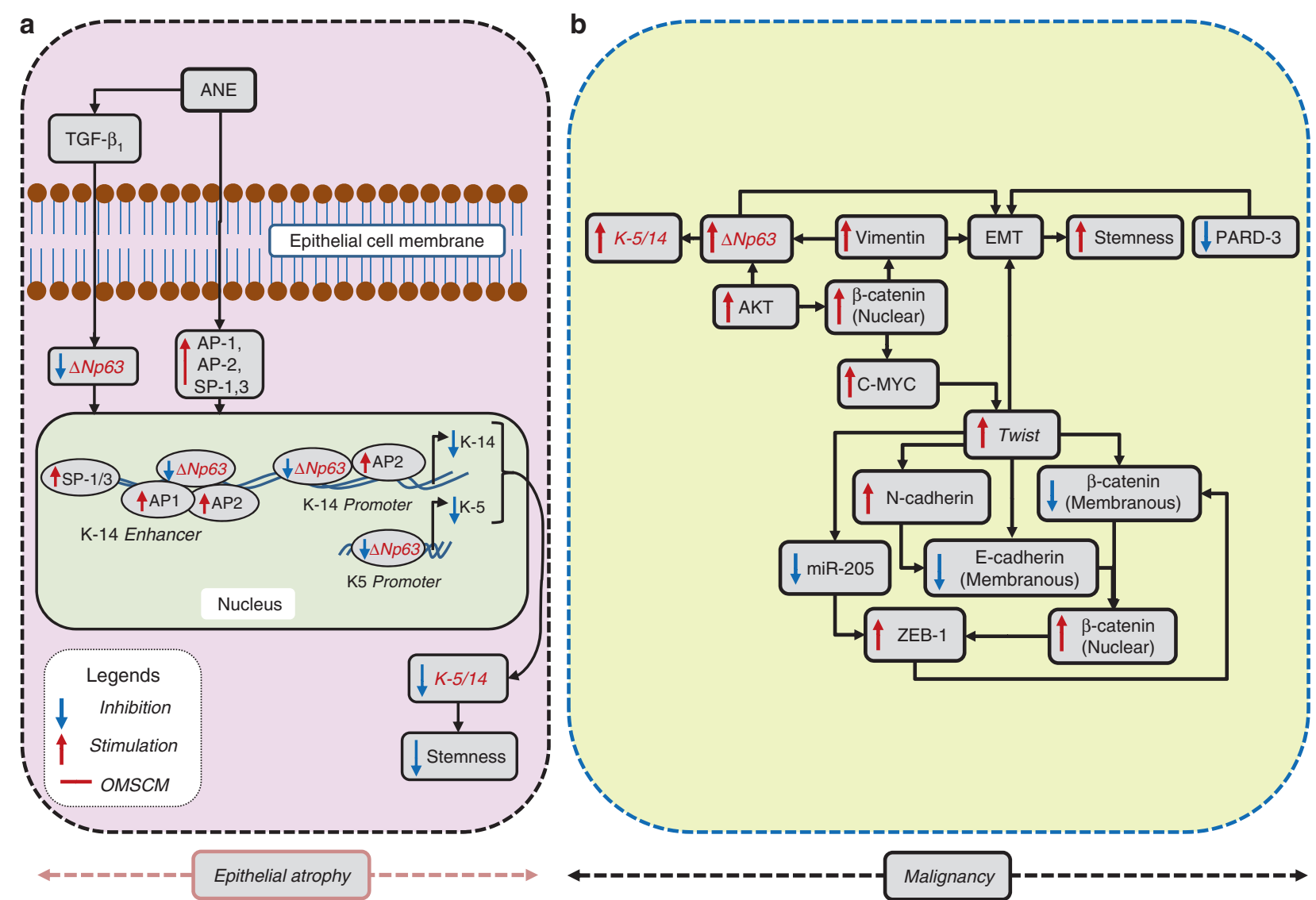

Fig. $4 \mathrm{~K}-5 / 14$ as an oral mucosal stem cell marker (OM-SCM) in oral submucous fibrosis (OSF). a Its downregulation mediates epithelial atrophy, and $\mathbf{b}$ its upregulation mediates malignancy

established that a higher coexpression of vimentin and $\mathrm{K}-14$ correlates with the recurrence and poor survival of OSCC (Fig. 4b). ${ }^{65}$

Keratin-19 as a stem cell marker in OSF and its malignant evolution

Keratin-19 expression, restricted to epithelial cells, is essential for the maintenance of the proliferative potential of the basal stem cell layer in nonkeratinized mucosa. Its loss correlates with the loss of self-renewal capacity and subsequent atrophy in OSF., ${ }^{899}$ The atrophic epithelium further facilitates fibrosis through enhanced permeability. ${ }^{8}$ High-throughput oligonucleotide microarray analysis has demonstrated $\mathrm{K}-19$ to be the topmost among 129 downregulated genes in OSF. ${ }^{8}$ Incidentally, K-19 expression shows an inverse correlation with arecoline concentration. ${ }^{99}$

Cytokeratin fragment antigen 21-1 (CYFRA21-1), a cytokeratin fragment produced through the action of caspase-3 on keratin-19, has been reported in the serum and saliva of patients with fibrosis, including OSF, as well as in those with malignancy. ${ }^{100}$ In vitro experiments have shown the cessation of CYFRA21-1 release into the culture supernatant with the addition of a caspase-3- specific inhibitor (Fig. 5a). ${ }^{101}$ Stromal hypoxia leading to caspase-3 and -9 activation is the most plausible explanation for the downregulation of K-19 and the appearance of CYFRA21-1 in OSF. ${ }^{102,103}$ CYFRA21-1 is thus a promising noninvasive proxy marker of hypoxia-initiated epithelial apoptosis and atrophy in OSF patients (Fig. 5a). ${ }^{101}$

While $\mathrm{K}-19$ is downregulated in OSF, it is upregulated in OED. ${ }^{100,104}$ Furthermore, it demonstrates a sequential increase with progressive grades of OED and OSCC. ${ }^{104,105}$ Moreover, the expression of K-19 is the highest at the invasive front, ${ }^{105}$ and serves as an independent predictor of poor prognosis. ${ }^{106}$ Although the mechanism for alterations in K-19 expression in the malignant transformation of OSF has not been studied, increased K-19 expression in OED, OSCC and malignant OSF could be mediated through enhanced matrix stiffness, which itself is due to progressive matrix cross-linking. The increased stiffness in OSF mucosa is evident by a thickened basement membrane, subepithelial fibrosis and increased collagen density. ${ }^{12}$ The enhanced matrix stiffness increases myofibroblast formation, which in turn intensifies collagen formation. ${ }^{2}$ The increased collagen bioavailability and its binding to discoid domain receptor-2 (DDR-2) enhances constitutive src activation. ${ }^{107}$ The upregulation of K-19 is due to the phosphorylation of its tyrosine 394 residue by the constitutive activation of src kinase, which otherwise is not phosphorylated in the basal state (Fig. 5b). ${ }^{108}$ This will lead to a complex phenotype of increased K-19 breakdown evidenced by CYFRA21-1 expression in saliva/serum with a superimposed upregulation of K-19 through a previously discussed fibronectinand/or type- 1 collagen $/ \beta_{1}$-integrin/Src-based mechanism.

Increasing matrix stiffness through a progressively cross-linked ECM might mediate the upregulation of Yes-associated protein 1 (YAP-1) and its transcriptional coactivator with PDZ-binding motif (TAZ) $^{109-111}$ in OSF. Interestingly, $\triangle$ Np63a forms a complex with YAP-1, which promotes stem cell survival. ${ }^{112}$ Moreover, YAP-1 can trigger SOX2 transcription by physical interaction with OCT-4. ${ }^{113}$ In addition, TAZ binds to the promoter of SOX2, which then drives stemness in oral cancer. ${ }^{113}$ YAP-1 upregulates CTGF (which induces proliferation) and C-MYC (which enhances clonogenicity), downregulates caspase- 7 and $\mathrm{BAX}$ and upregulates $\mathrm{BCl}-2$ (which enhances apoptosis resistance) (Fig. 5b). ${ }^{114}$ These mechanisms might be relevant to the malignant transformation of OSF. 


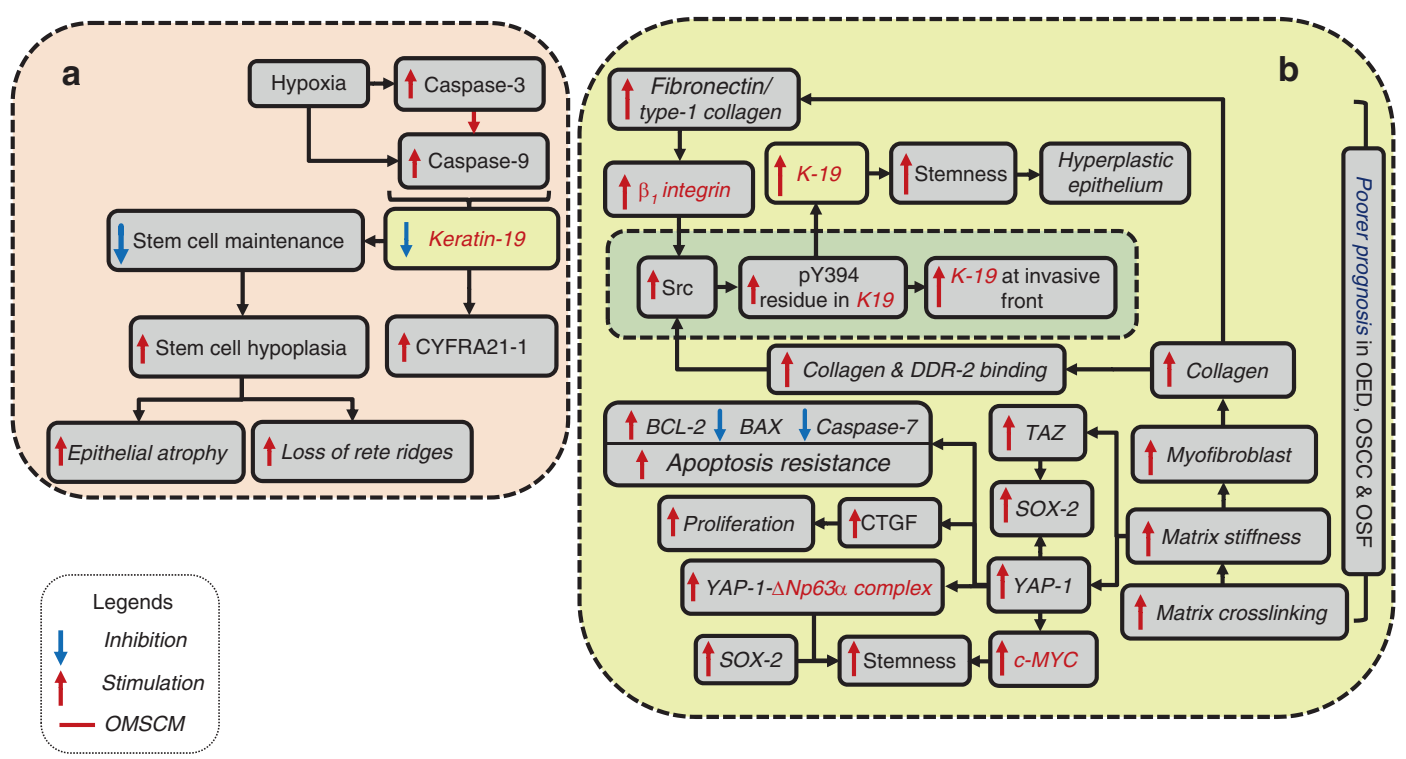

Fig. 5 K-19 as an oral mucosal stem cell marker (OM-SCM) in oral submucous fibrosis (OSF). a Its downregulation mediates epithelial atrophy, and $\mathbf{b}$ its upregulation mediates malignancy

\section{RISE OF CANCER STEM CELLS-DICHOTOMY OF ARECA NUT INGREDIENTS IN EPITHELIAL AND STROMAL COMPARTMENTS} The origin of CSCs in OSCCs has been attributable ${ }^{115}$ to various factors, which are described as follows.

The genetic alterations in the basal epithelial stem cells It has been experimentally demonstrated that $\mathrm{CD} 44^{+}$is expressed in the basal cell compartment and absent in differentiated cells. ${ }^{26}$ The protracted contact of arecoline with oral keratinocytes leads to the upregulation of various stem cell markers, such as ALDH1, CD44, Nanog, Oct-4 and SOX2. ${ }^{18}$

Upregulation of EMT in epithelial cells

The chronic use of areca nut leads to widespread effects, such as autophagy induction, promotion of EMT and increased stemness, which then promote oral cancer. ${ }^{18} \mathrm{CSCs}$ are a plastic state of tumour cells undergoing EMT. Approximately $40-50 \%$ of oral cancer recurrence is attributed to EMT. Lazarevic et al. ${ }^{39}$ stated that EMT preferentially occurs at tumour margins, as they are sites of vessel invasion. The expression of EMT markers tended to be higher in surgical margins than within tumours. ${ }^{39}$

Oral cancer cells expressing SNAI1 (a master mediator of EMT) acquire a CSC-like phenotype, chemoresistance and migration and invasion potential. ${ }^{45}$ In cells expressing both $\mathrm{ALDH} 1^{+}$and $\mathrm{CD} 44^{+}$, Snail coexpresses with ALDH1. Its experimental suppression decreases the expression of ALDH1, inhibits CSC-like properties and decreases tumorigenic potential. This suggests that in $\mathrm{ALDH} 1^{+}$cells, CSC properties are mediated by Snail, and that its reversal reduces chemoresistance. ${ }^{43}$

Epithelial stemness augmentation by mesenchymal stem cells Resident and bone marrow-derived mesenchymal stem cells (MSCs) are precursors of stroma associated with cancer. ${ }^{29}$ These stem cells contribute to angiogenesis and lymphangiogenesis, modulate the immune system and produce tumour-associated myofibroblasts. $^{29}$ In OSCC, fibroblasts can be activated into myofibroblasts either through genetically transformed keratinocytes or exogenous agents such as irradiation or viruses. ${ }^{28}$ This in turn can stimulate the transformed keratinocytes by influencing stem cell division patterns towards symmetry, with an increase in the stem cell pool within the lesion. ${ }^{28}$

Via TGF- $\beta$, arecoline drives the upregulation of the mesenchymal stem cell (MSC) marker STRO-1 in OSF. ${ }^{116}$ The increased conversion of fibroblasts into myofibroblasts and upregulated stemness are indicated by their increased contraction, migration, invasion and expression of a-SMA and the pro a- 1 chain of type- 1 collagen. ${ }^{116}$ These MSCs can serve as a source of multipotent cells that can rapidly repopulate the wound in response to epithelial injury. ${ }^{116}$

Ye et al. ${ }^{117}$ showed that growth-regulated oncogene-a (GRO-a) secretion by OSF fibroblasts promotes oral keratinocyte malignant transformation by augmenting EGFR/ERK signalling, F-actin rearrangement and stemness. Transformed keratinocytes can then acquire further genetic alterations with the evolution of more invasive clones. Highly motile myofibroblasts may also come into close contact with the highly spindled transformed stem cells and fuse to produce a more aggressive cell with the myofibroblast property of high motility and the stem cell property of high selfrenewal. $^{28}$

Thus, there seems to be a dichotomy with respect to epithelial and mesenchymal clonogenicity in OSF in response to chronic areca nut chewing. Interestingly, the increased stemness of the stromal cell compartment drives a secondary increase in epithelial stemness, which could be an important mechanism of malignant transformation in OSF.

\section{FUTURE PERSPECTIVES}

Various molecular pathways (Figs. 1-5) and pervasive epithelial-stromal interactions underlie the mechanisms of the upregulation of epithelial stem cells and their role in malignancy. Practically, the normal buccal mucosal epithelium, with an intact basal stem cell layer, has the potential to ameliorate fibrosis through the recompensation of basal stem activity. The restoration of the basal stem cell layer via stem cell therapy should be considered an important component of OSF therapy. 4,8

Recent studies have demonstrated the importance of the perivascular stem cell niche in oral cancer. It is present in the ITF where CSCs reside, in close proximity to the blood vessels. Endothelial cell-initiated signalling has been shown to be critical for the survival and self-renewal of CSCs, and may play a role in resistance to therapy. Therefore, oral cancer patients might benefit from therapies that target CSCs directly or their supportive perivascular niche. ${ }^{18,119}$

Newly designed chemotherapy and radiotherapy regimens for the treatment of solid cancers have failed to improve patient 
survival. The underlying reason for this therapeutic failure is that these regimens target fast-dividing cells instead of slow-dividing CSCs. ${ }^{120-123}$ The slow cycling and constitutive expression of multiple members of the ATP-binding cassette (ABC) family of transporters is responsible for tumour stem cells exhibiting a high degree of chemoresistance. ${ }^{124,125}$ Thus, the final therapeutic goal should be to restore epithelial homoeostasis. Reinstating the renewal ability of the basal layer of oral mucosa should reverse fibrosis and prevent malignancy. ${ }^{2,8,126-129}$ Indeed, the coculture of normal buccal mucosal epithelial cell fibrotic fibroblasts causes the downregulation of connective tissue growth factor (CTGF), ${ }^{128}$ a profibrotic mediator in OSF. ${ }^{2}$ The undifferentiated keratinocytes reinstate the normal healing patterns in fibrosis through the downregulation of TGF- $\beta$ and the stabilization of desmosomal assembly. ${ }^{129}$ Targeting the reactive tumour-stroma could be another approach to halt the signals from the microenvironment that prevent stem cell recovery. ${ }^{28,31}$

\section{CONCLUSION}

There is compelling evidence to suggest that the aberrant selfrenewing capacity of the basal stem cell layer in OSF with atrophic epithelium and its restitution in hyperplastic and/or dysplastic epithelium are associated with the progression to malignancy. Tumour-stroma interactions provide a niche to potentiate cancer stem cell behaviour, and the tumour-associated stroma has been shown to revive basal stem cell activity by initiating a vicious cycle between epithelial and stromal stem cell compartments.

It is possible that a reduction in stem cell activity causes epithelial atrophy in OSF, and its restitution promotes malignancy. The vacillating expression of keratin $5,14,19, C D 44, \beta 1$-integrin, p63, Oct-4, c-MYC, c-MET and ALDH1 supports this hypothesis. There are no reports highlighting an oscillating relationship of OM-SCMs in atrophic OSF versus OPMDs and OSCCs. How the restitution of stem cell activity contributes to malignant transformation has been schematically illustrated for the first time. Furthermore, the role of OM-SCMs in atrophic OSF versus hyperplastic OSF, OED and OSCC is a fertile area of research, which may provide further credence in the treatment and management of OSF.

\section{ACKNOWLEDGEMENTS}

This work was supported by the Science and Engineering Research Board (SERB), Department of Science and Technology and Government of India sanction order No. EMR/2017/002792 dated 25 September 2018 for the research project entitled "Characterization of novel TGF- $\beta 1 /$ SMAD regulated microRNAs in the rat model of oral submucous fibrosis and the effect of Resveratrol as an Anti-Fibrotic Agent".

\section{AUTHOR CONTRIBUTIONS}

M.S. and R.R. developed the study concepts and design. M.S. and R.R. prepared the schemas and acquired data. M.S., K.D.H. and F.P.F. prepared the initial manuscript preparation; F.P.F., R.R. and K.D.H. edited the manuscript reviewed.

\section{ADDITIONAL INFORMATION}

The online version of this article (https://doi.org/10.1038/s41368-020-00090-5) contains supplementary material, which is available to authorized users.

Competing interests: The authors declare no competing interests.

\section{REFERENCES}

1. Ray, J. G., Chatterjee, R. \& Chaudhuri, K. Oral submucous fibrosis: a global challenge. Rising incidence, risk factors, management, and research priorities. Periodontol 2000 80, 200-212 (2019).
2. Sharma, M., Shetty, S. S. \& Radhakrishnan, R. Oral submucous fibrosis as an overhealing wound: implications in malignant transformation. Recent Pat. Anticancer Drug Discov. 13, 272-291 (2018).

3. Shih, Y. H., Wang, T. H., Shieh, T. M. \& Tseng, Y. H. Oral submucous fibrosis: a review on etiopathogenesis, diagnosis, and therapy. Int. J. Mol. Sci. 20, 2940 (2019).

4. Khan, I. et al. Epithelial atrophy in oral submucous fibrosis is mediated by copper (II) and arecoline of areca nut. J. Cell. Mol. Med. 19, 2397-2412 (2015).

5. Jones, R. E., Foster, D. S., Hu, M. S. \& Longaker, M. T. Wound healing and fibrosis: current stem cell therapies. Transfusion 59, 884-892 (2019).

6. Ge, Y. et al. Stem cell lineage infidelity drives wound repair and cancer. Cell 169, 636-650 (2017).

7. Rajendran, R., Sunil, Twinkle, S. P., Anikumar, T. V. \& Annie, J. Cell death does not herald epithelial involution ("atrophy") in oral sub mucous fibrosis: a TEM study. Indian J. Dent. Res. 15, 13-19 (2004).

8. Li, N. et al. Discovery of novel biomarkers in oral submucous fibrosis by microarray analysis. Cancer Epidemiol. Biomark. Prev. 17, 2249-2259 (2008).

9. Veeravarmal, V., Austin, R. D., Nagini, S. \& Nassar, M. H. M. Expression of $\beta 1$ integrin in normal epithelium, oral submucous fibrosis and oral squamous cell carcinoma. Pathol. Res. Pract. 214, 273-280 (2018).

10. Bazarsad, S. et al. Identification of a combined biomarker for malignant transformation in oral submucous fibrosis. J. Oral. Pathol. Med. 46, 431-438 (2017).

11. Rajendran, R. \& Varkey, S. Inducible nitric oxide synthase expression is upregulated in oral submucous fibrosis. Indian J. Dent. Res. 18, 94-100 (2007).

12. Das, R. K. et al. Epithelio-mesenchymal transitional attributes in oral sub-mucous fibrosis. Exp. Mol. Pathol. 95, 259-269 (2013).

13. Anura, A. et al. Endorsing cellular competitiveness in aberrant epithelium of oral submucous fibrosis progression: neighbourhood analysis of immunohistochemical attributes. Histochem. Cell Biol. 150, 61-75 (2018).

14. Das, R. K. et al. Assessment of malignant potential of oral submucous fibrosis through evaluation of p63, E-cadherin and CD105 expression. J. Clin. Pathol. 63, 894-899 (2010).

15. Overstreet, J. M., Samarakoon, R., Meldrum, K. K. \& Higgins, P. J. Redox control of p53 in the transcriptional regulation of TGF-beta1 target genes through SMAD cooperativity. Cell Signal. 26, 1427-1436 (2014).

16. Kawarada, Y. et al. TGF- $\beta$ induces $\mathrm{p} 53 / \mathrm{Smads}$ complex formation in the PAI-1 promoter to activate transcription. Sci. Rep. 6, 35483 (2016).

17. Sachdeva, M. et al. p53 represses c-Myc through induction of the tumor suppressor miR-145. Proc. Natl Acad. Sci. USA 106, 3207-3212 (2009).

18. Wang, T. Y. et al. Acquisition cancer stemness, mesenchymal transdifferentiation, and chemoresistance properties by chronic exposure of oral epithelial cells to arecoline. Oncotarget 7, 84072-84081 (2016).

19. Baillie, R., Tan, S. T. \& Itinteang, T. Cancer stem cells in oral cavity squamous cell carcinoma: a review. Front. Oncol. 7, 112 (2017).

20. Feng, J. Q. et al. Expression of cancer stem cell markers ALDH1 and Bmi1 in oral erythroplakia and the risk of oral cancer. J. Oral. Pathol. Med. 42, 148-153 (2013).

21. Rodini, C. O., Lopes, N. M., Lara, V. S. \& Mackenzie, I. C. Oral cancer stem cellsproperties and consequences. J. Appl. Oral. Sci. 25, 708-715 (2017).

22. Jones, K. B. \& Klein, O. D. Oral epithelial stem cells in tissue maintenance and disease: the first steps in a long journey. Int. J. Oral. Sci. 5, 121-129 (2013).

23. Batlle, E. \& Clevers, H. Cancer stem cells revisited. Nat. Med. 23, 1124-1134 (2017).

24. Rich, J. N. Cancer stem cells: understanding tumor hierarchy and heterogeneity. Medicine 95, S2-S7 (2016).

25. Hu, F. W. et al. Impairment of tumor-initiating stem-like property and reversal of epithelial-mesenchymal transdifferentiation in head and neck cancer by resveratrol treatment. Mol. Nutr. Food Res. 56, 1247-1258 (2012).

26. Sinha, N., Mukhopadhyay, S., Das, D. N., Panda, P. K. \& Bhutia, S. K. Relevance of cancer initiating/stem cells in carcinogenesis and therapy resistance in oral cancer. Oral. Oncol. 49, 854-862 (2013).

27. Chen, Y. C. et al. Inhibition of tumorigenicity and enhancement of radiochemosensitivity in head and neck squamous cell cancer-derived ALDH1-positive cells by knockdown of Bmi-1. Oral. Oncol. 46, 158-165 (2010).

28. Costea, D. E., Tsinkalovsky, O., Vintermyr, O. K., Johannessen, A. C. \& Mackenzie, I. C. Cancer stem cells-new and potentially important targets for the therapy of oral squamous cell carcinoma. Oral. Dis. 12, 443-454 (2006).

29. De Boeck, A. et al. Resident and bone marrow-derived mesenchymal stem cells in head and neck squamous cell carcinoma. Oral. Oncol. 46, 336-342 (2010).

30. Schneider, S. et al. Expression of the sonic hedgehog pathway in squamous cell carcinoma of the skin and the mucosa of the head and neck. Head. Neck 33, 244-250 (2011).

31. Prince, M. E. et al. Identification of a subpopulation of cells with cancer stem cell properties in head and neck squamous cell carcinoma. Proc. Natl Acad. Sci. USA 104, 973-978 (2007). 
32. Fu, T. Y. et al. Association of OCT4, SOX2, and NANOG expression with oral squamous cell carcinoma progression. J. Oral. Pathol. Med. 45, 89-95 (2016).

33. Rodrigues, M. et al. Prognostic implications of CD44, NANOG, OCT4, and BMI1 expression in tongue squamous cell carcinoma. Head Neck 40, 1759-1773 (2018).

34. Ortiz, R. C. et al. CD44 and ALDH1 immunoexpression as prognostic indicators of invasion and metastasis in oral squamous cell carcinoma. J. Oral. Pathol. Med. 47, 740-747 (2018).

35. Lobo, N. A., Shimono, Y., Qian, D. \& Clarke, M. F. The biology of cancer stem cells. Annu. Rev. Cell Dev. Biol. 23, 675-699 (2007).

36. Katoh, M. \& Katoh, M. WNT signaling pathway and stem cell signaling network. Clin. Cancer Res. 13, 4042-4045 (2007).

37. Milosevic, M. et al. Characterization of stem-like cancer cells in basal cell carcinoma and its surgical margins. Exp. Dermatol. 27, 1160-1165 (2018).

38. Lazarevic, M. et al. Putative cancer stem cells are present in surgical margins of oral squamous cell carcinoma. J. BUON 23, 1686-1692 (2018).

39. Lazarevic, M. et al. Marked epithelial to mesenchymal transition in surgical margins of oral canceran in vitro study. Oncol. Lett. 19, 3743-3750 (2020).

40. Kobayashi, C. I. \& Suda, T. Regulation of reactive oxygen species in stem cells and cancer stem cells. J. Cell. Physiol. 227, 421-430 (2012).

41. Xu, Q. et al. EGF induces epithelial-mesenchymal transition and cancer stem-like cell properties in human oral cancer cells via promoting Warburg effect. Oncotarget 8, 9557-9571 (2017).

42. Zhao, H., Hu, C. Y., Chen, W. M. \& Huang, P. Lactate promotes cancer stem-like property of oral sequamous cell carcinoma. Curr. Med. Sci. 39, 403-409 (2019).

43. Chen, Y. C. et al. Aldehyde dehydrogenase 1 is a putative marker for cancer stem cells in head and neck squamous cancer. Biochem. Biophys. Res. Commun. 385, 307-313 (2009).

44. Bertrand, G. et al. Targeting head and neck cancer stem cells to overcome resistance to photon and carbon ion radiation. Stem Cell Rev. 10, 114-126 (2014).

45. Masui, T. et al. Snail-induced epithelial-mesenchymal transition promotes cancer stem cell-like phenotype in head and neck cancer cells. Int. J. Oncol. 44, 693-699 (2014).

46. Seino, S. et al. CD44(high) /ALDH1(high) head and neck squamous cell carcinoma cells exhibit mesenchymal characteristics and GSK3beta-dependent cancer stem cell properties. J. Oral. Pathol. Med. 45, 180-188 (2016).

47. Hildebrand, L. C. et al. Spatial distribution of cancer stem cells in head and neck squamous cell carcinomas. J. Oral. Pathol. Med. 43, 499-506 (2014).

48. Visus, $C$. et al. Identification of human aldehyde dehydrogenase 1 family member $\mathrm{A} 1$ as a novel CD8+ T-cell-defined tumor antigen in squamous cell carcinoma of the head and neck. Cancer Res. 67, 10538-10545 (2007).

49. Li, Y. C., Cheng, A. J., Lee, L. Y., Huang, Y. C. \& Chang, J. T. Multifaceted mechanisms of areca nuts in oral carcinogenesis: the molecular pathology from precancerous condition to malignant transformation. J. Cancer 10, 4054-4062 (2019).

50. Li, Y. C. et al. Areca nut contributes to oral malignancy through facilitating the conversion of cancer stem cells. Mol. Carcinog. 55, 1012-1023 (2016).

51. Liu, W. et al. Two stem cell markers, ATP-binding cassette, G2 subfamily (ABCG2) and BMI-1, predict the transformation of oral leukoplakia to cancer: a long-term follow-up study. Cancer 118, 1693-1700 (2012).

52. Liu, W. et al. Expression patterns of cancer stem cell markers ALDH1 and CD133 correlate with a high risk of malignant transformation of oral leukoplakia. Int. J. Cancer 132, 868-874 (2013).

53. Feng, J., Zhou, Z., Shi, L., Yang, X. \& Liu, W. Cancer stem cell markers ALDH1 and Bmi1 expression in oral erythroplakia revisited: implication for driving the process of field cancerization. J. Oral. Pathol. Med. 49, 96-99 (2020).

54. Yang, X., Shi, L., Zhou, Z. \& Liu, W. Podoplanin and ABCG2 expression in oral erythroplakia revisited: potential evidence for cancer stem cells driving the process of field cancerization. Oral. Oncol. 101, 104368 (2020).

55. Calenic, B., Greabu, M., Caruntu, C., Tanase, C. \& Battino, M. Oral keratinocyte stem/progenitor cells: specific markers, molecular signaling pathways and potential uses. Periodontol 2000 69, 68-82 (2015).

56. Li, N., Li, X., Chen, K., Dong, H. \& Kagami, H. Characterization of spontaneous spheroids from oral mucosa-derived cells and their direct comparison with spheroids from skin-derived cells. Stem Cell Res. Ther. 10, 184 (2019).

57. Hisha, H., Tanaka, T. \& Ueno, H. Lingual epithelial stem cells and organoid culture of them. Int. J. Mol. Sci. 17, 168 (2016).

58. Moses, M. A. et al. Molecular mechanisms of p63-mediated squamous cancer pathogenesis. Int. J. Mol. Sci. 20, 3590 (2019).

59. Bose, A. et al. Two mechanisms regulate keratin K15 expression in keratinocytes: role of PKC/AP-1 and FOXM1 mediated signalling. PLOS ONE 7, e38599 (2012).

60. Ishii, A. et al. Expression of p75(NGFR), a proliferative and basal cell marker, in the buccal mucosa epithelium during re-epithelialization. Acta Histochem. Cytochem. 47, 145-153 (2014).
61. Cherukuri, P. et al. Phosphorylation of DeltaNp63alpha via a novel TGFbeta/ ALK5 signaling mechanism mediates the anti-clonogenic effects of TGFbeta. PLOS ONE 7, e50066 (2012).

62. Hildesheim, J. et al. Gadd45a regulates matrix metalloproteinases by suppressing $\triangle \mathrm{Np} 63 \mathrm{a}$ and $\beta$-catenin via p38 MAP kinase and APC complex activation. Oncogene 23, 1829-1837 (2004).

63. Cojoc, M. et al. Aldehyde dehydrogenase is regulated by $\beta$-catenin/TCF and promotes radioresistance in prostate cancer progenitor cells. Cancer Res. 75, 1482-1494 (2015)

64. Alam, H., Sehgal, L., Kundu, S. T., Dalal, S. N. \& Vaidya, M. M. Novel function of keratins 5 and 14 in proliferation and differentiation of stratified epithelial cells. Mol. Biol. Cell 22, 4068-4078 (2011).

65. Dmello, C. et al. Vimentin regulates differentiation switch via modulation of keratin 14 levels and their expression together correlates with poor prognosis in oral cancer patients. PLOS ONE 12, e0172559 (2017).

66. Xia, $\mathrm{H}$. et al. Pathologic caveolin-1 regulation of PTEN in idiopathic pulmonary fibrosis. Am. J. Pathol. 176, 2626-2637 (2010).

67. Lin, H. C. et al. High-level beta1-integrin expression in a subpopulation of highly tumorigenic oral cancer cells. Clin. Oral. Investig. 18, 1277-1284 (2014).

68. Barkan, D. \& Chambers, A. F. $\beta 1$-integrin: a potential therapeutic target in the battle against cancer recurrence. Clin. Cancer Res. 17, 7219-7223 (2011).

69. Shishido, S., Bonig, H. \& Kim, Y. M. Role of integrin alpha4 in drug resistance of leukemia. Front. Oncol. 4, 99 (2014).

70. Zhang, H. F. et al. The PI3K/AKT/c-MYC axis promotes the acquisition of cancer stem-like features in esophageal squamous cell carcinoma. Stem Cells 34, 2040-2051 (2016).

71. Ha, L., Ponnamperuma, R. M., Jay, S., Ricci, M. S. \& Weinberg, W. C. Dysregulated DeltaNp63alpha inhibits expression of Ink4a/arf, blocks senescence, and promotes malignant conversion of keratinocytes. PLoS ONE 6, e21877 (2011).

72. Chang, M. C. et al. Areca nut components affect COX-2, cyclin B1/cdc25C and keratin expression, PGE2 production in keratinocyte is related to reactive oxygen species, CYP1A1, Src, EGFR and Ras signaling. PLoS ONE 9, e101959 (2014).

73. Mognetti, B. et al. $\triangle \mathrm{Np} 63 \mathrm{a}$ as early indicator of malignancy in surgical margins of an oral squamous cell carcinoma. Oral. Oncol. Extras 41, 129-131 (2005).

74. Candi, E. et al. TAp63 and DeltaNp63 in cancer and epidermal development. Cell Cycle 6, 274-285 (2007).

75. Wang, G. X. et al. DeltaNp63 inhibits oxidative stress-induced cell death, including ferroptosis, and cooperates with the BCL-2 family to promote clonogenic survival. Cell Rep. 21, 2926-2939 (2017).

76. Compagnone, M. et al. DeltaNp63-mediated regulation of hyaluronic acid metabolism and signaling supports HNSCC tumorigenesis. Proc. Natl Acad. Sci. USA 114, 13254-13259 (2017).

77. Keyes, W. M. et al. DeltaNp63alpha is an oncogene that targets chromatin remodeler Lsh to drive skin stem cell proliferation and tumorigenesis. Cell Stem Cell 8, 164-176 (2011).

78. Viticchie, G. et al. p63 supports aerobic respiration through hexokinase II. Proc. Natl Acad. Sci. USA 112, 11577-11582 (2015).

79. Roberts, D. J. \& Miyamoto, S. Hexokinase II integrates energy metabolism and cellular protection: akting on mitochondria and TORCing to autophagy. Cell Death Differ. 22, 248-257 (2015).

80. Victorelli, S. \& Passos, J. F. Reactive oxygen species detection in senescent cells. Methods Mol. Biol. 1896, 21-29 (2019).

81. He, X. et al. Chromatin remodeling factor LSH drives cancer progression by suppressing the activity of fumarate hydratase. Cancer Res. 76, 5743-5755 (2016).

82. Yang, M., Soga, T., Pollard, P. J. \& Adam, J. The emerging role of fumarate as an oncometabolite. Front. Oncol. 2, 85-85 (2012).

83. Courtnay, R. et al. Cancer metabolism and the Warburg effect: the role of HIF-1 and PI3K. Mol. Biol. Rep. 42, 841-851 (2015).

84. Bourguignon, L. Y. W. Matrix hyaluronan-CD44 interaction activates MicroRNA and LncRNA signaling associated with chemoresistance, invasion, and tumor progression. Front. Oncol. 9, 492 (2019).

85. Yoh, K. \& Prywes, R. Pathway regulation of p63, a director of epithelial cell fate. Front. Endocrinol. 6, 51 (2015).

86. Chu, W. K., Dai, P. M., Li, H. L. \& Chen, J. K. Transcriptional activity of the DeltaNp63 promoter is regulated by STAT3. J. Biol. Chem. 283, 7328-7337 (2008).

87. Wong, W. J., Qiu, B., Nakazawa, M. S., Qing, G. \& Simon, M. C. MYC degradation under low $\mathrm{O} 2$ tension promotes survival by evading hypoxia-induced cell death. Mol. Cell. Biol. 33, 3494-3504 (2013).

88. Wang, L., Xue, M. \& Chung, D. C. c-Myc is regulated by HIF-2alpha in chronic hypoxia and influences sensitivity to 5 -FU in colon cancer. Oncotarget 7 , 78910-78917 (2016).

89. Wang, L. H., Xu, M., Fu, L. Q., Chen, X. Y. \& Yang, F. The antihelminthic niclosamide inhibits cancer stemness, extracellular matrix remodeling, and 
metastasis through dysregulation of the nuclear beta-catenin/c-Myc axis in OSCC. Sci. Rep. 8, 12776 (2018).

90. Kim, M. J. et al. PAF-Myc-controlled cell stemness is required for intestinal regeneration and tumorigenesis. Dev. Cell 44, 582-596 (2018). e4.

91. Huang, H. et al. NF-kappaB1 inhibits C-Myc protein degradation through suppression of FBW7 expression. Oncotarget 5, 493-505 (2014).

92. Sawant, S. et al. Prognostic role of Oct4, CD44 and C-Myc in radio-chemoresistant oral cancer patients and their tumourigenic potential in immunodeficient mice. Clin. Oral. Investig. 20, 43-56 (2016).

93. Itahana, K., Campisi, J. \& Dimri, G. P. Mechanisms of cellular senescence in human and mouse cells. Biogerontology 5, 1-10 (2004).

94. Jacobs, J. J., Kieboom, K., Marino, S., DePinho, R. A. \& van Lohuizen, M. The oncogene and polycomb-group gene bmi-1 regulates cell proliferation and senescence through the ink4a locus. Nature 397, 164-168 (1999).

95. Kang, M. K. et al. Elevated Bmi-1 expression is associated with dysplastic cell transformation during oral carcinogenesis and is required for cancer cell replication and survival. Br. J. Cancer 96, 126-133 (2007).

96. Nacerddine, K. et al. Akt-mediated phosphorylation of Bmi1 modulates its oncogenic potential, E3 ligase activity, and DNA damage repair activity in mouse prostate cancer. J. Clin. Investig. 122, 1920-1932 (2012).

97. $\mathrm{Yu}, \mathrm{C}$. C. et al. Bmi-1 regulates snail expression and promotes metastasis ability in head and neck squamous cancer-derived ALDH1 positive cells. J. Oncol. 2011, 609259 (2011).

98. Sawant, S. S. et al. Clinical significance of aberrant vimentin expression in oral premalignant lesions and carcinomas. Oral. Dis. 20, 453-465 (2014).

99. Zheng, L. et al. miR-203 inhibits arecoline-induced epithelial-mesenchymal transition by regulating secreted frizzled-related protein 4 and transmembrane$4 \mathrm{~L}$ six family member 1 in oral submucous fibrosis. Oncol. Rep. 33, 2753-2760 (2015).

100. Rajkumar, K. et al. Salivary and serum level of CYFRA 21-1 in oral precancer and oral squamous cell carcinoma. Oral. Dis. 21, 90-96 (2015).

101. Dohmoto, K. et al. The role of caspase 3 in producing cytokeratin 19 fragment (CYFRA21-1) in human lung cancer cell lines. Int. J. Cancer 91, 468-473 (2001).

102. Garnier, P. et al. Hypoxia induces caspase-9 and caspase-3 activation without neuronal death in gerbil brains. Eur. J. Neurosci. 20, 937-946 (2004).

103. Veeravarmal, V., Austin, R. D., Siddavaram, N., Thiruneelakandan, S. \& Nassar, M. H. Caspase-3 expression in normal oral epithelium, oral submucous fibrosis and oral squamous cell carcinoma. J. Oral. Maxillofac. Pathol. 20, 445-452 (2016).

104. Safadi, R. A., Musleh, A. S., Al-Khateeb, T. H. \& Hamasha, A. A. Analysis of immunohistochemical expression of $\mathrm{k} 19$ in oral epithelial dysplasia and oral squamous cell carcinoma using color deconvolution-image analysis method. Head. Neck Pathol. 4, 282-289 (2010).

105. Khanom, R. et al. Expression of basal cell keratin 15 and keratin 19 in oral squamous neoplasms represents diverse pathophysiologies. Histol. Histopathol. 27, 949-959 (2012).

106. Ernst, J. et al. Expression of CK19 is an independent predictor of negative outcome for patients with squamous cell carcinoma of the tongue. Oncotarget 7, 76151-76158 (2016).

107. Carafoli, F. \& Hohenester, E. Collagen recognition and transmembrane signalling by discoidin domain receptors. Biochim. Biophys. Acta 1834, 2187-2194 (2013).

108. Zhou, Q. et al. Characterization of in vivo keratin 19 phosphorylation on tyrosine391. PLOS ONE 5, e13538 (2010).

109. Matte, B. F. et al. Matrix stiffness mechanically conditions EMT and migratory behavior of oral squamous cell carcinoma. J. Cell Sci. 132, jcs224360 (2019).

110. Hiemer, S. E. et al. A YAP/TAZ-regulated molecular signature is associated with oral squamous cell carcinoma. Mol. Cancer Res. 13, 957-968 (2015).

111. Nukuda, A. et al. Stiff substrates increase YAP-signaling-mediated matrix metalloproteinase-7 expression. Oncogenesis 4, e165 (2015).
112. Fisher, M. L., Ciavattone, N., Grun, D., Adhikary, G. \& Eckert, R. L. Sulforaphane reduces YAP/Np63alpha signaling to reduce cancer stem cell survival and tumor formation. Oncotarget 8, 73407-73418 (2017).

113. Li, J. et al. The hippo effector TAZ promotes cancer stemness by transcriptional activation of SOX2 in head neck squamous cell carcinoma. Cell Death Dis. 10, 603 (2019).

114. Chen, X. et al. C-MYC and BCL-2 mediate YAP-regulated tumorigenesis in OSCC. Oncotarget 9, 668-679 (2018).

115. Zhou, Z. T. \& Jiang, W. W. Cancer stem cell model in oral squamous cell carcinoma. Curr. Stem Cell Res. Ther. 3, 17-20 (2008).

116. Yu, C. C., Liao, Y. W., Yu, C. H. \& Chang, Y. C. STRO-1 confers myofibroblast transdifferentiation in fibroblasts derived from oral submucous fibrosis. J. Oral. Pathol. Med. 47, 299-305 (2018).

117. Ye, M. Y. et al. Growth-regulated oncogene-alpha from oral submucous fibrosis fibroblasts promotes malignant transformation of oral precancerous cells. $J$. Oral. Pathol. Med. 47, 880-886 (2018).

118. Ritchie, K. E. \& Nor, J. E. Perivascular stem cell niche in head and neck cancer. Cancer Lett. 338, 41-46 (2013).

119. Campos, M. S., Neiva, K. G., Meyers, K. A., Krishnamurthy, S. \& Nor, J. E. Endothelial derived factors inhibit anoikis of head and neck cancer stem cells. Oral. Oncol. 48, 26-32 (2012).

120. Winquist, R. J., Boucher, D. M., Wood, M. \& Furey, B. F. Targeting cancer stem cells for more effective therapies: taking out cancer's locomotive engine. Biochem. Pharmacol. 78, 326-334 (2009).

121. Ishii, H. et al. Cancer stem cells and chemoradiation resistance. Cancer Sci. 99, 1871-1877 (2008).

122. Frank, N. Y., Schatton, T. \& Frank, M. H. The therapeutic promise of the cancer stem cell concept. J. Clin. Investig. 120, 41-50 (2010).

123. Trumpp, A. \& Wiestler, O. D. Mechanisms of disease: cancer stem cells-targeting the evil twin. Nat. Clin. Pract. Oncol. 5, 337-347 (2008).

124. Glavinas, H., Krajcsi, P., Cserepes, J. \& Sarkadi, B. The role of ABC transporters in drug resistance, metabolism and toxicity. Curr. Drug Deliv. 1, 27-42 (2004).

125. Hatina, J. et al. Tumour stem cells-a new concept in tumour biology. Dtsch. Med. Wochenschr. 132, 1629-1632 (2007).

126. Epa, A. P. et al. Normal human lung epithelial cells inhibit transforming growth factor-beta induced myofibroblast differentiation via prostaglandin E2. PLOS ONE 10, e0135266 (2015).

127. Varga, J. \& Greten, F. R. Cell plasticity in epithelial homeostasis and tumorigenesis. Nat. Cell Biol. 19, 1133-1141 (2017).

128. Gottipamula, S., Sundarrajan, S., Moorthy, A., Padmanabhan, S. \& Sridhar, N. K. Buccal mucosal epithelial cells downregulate CTGF expression in buccal submucosal fibrosis fibroblasts. J. Maxillofac. Oral. Surg. 17, 254-259 (2018).

129. Wang, X. et al. Inhibition of dermal fibrosis in self-assembled skin equivalents by undifferentiated keratinocytes. J. Dermatol. Sci. 53, 103-111 (2009).

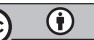

Open Access This article is licensed under a Creative Commons Attribution 4.0 International License, which permits use, sharing, adaptation, distribution and reproduction in any medium or format, as long as you give appropriate credit to the original author(s) and the source, provide a link to the Creative Commons license, and indicate if changes were made. The images or other third party material in this article are included in the article's Creative Commons license, unless indicated otherwise in a credit line to the material. If material is not included in the article's Creative Commons license and your intended use is not permitted by statutory regulation or exceeds the permitted use, you will need to obtain permission directly from the copyright holder. To view a copy of this license, visit http://creativecommons. org/licenses/by/4.0/.

(c) The Author(s) 2020 